\title{
Structure of the Hardy Operator Related to Laguerre Polynomials and the Euler Differential Equation
}

\author{
Natan KRUGlYAK, Lech MaligRANDA, \\ and Lars-Erik PERSSON \\ Department of Mathematics \\ Luleå University of Technology \\ SE-971 87 Luleå — Sweden \\ natan@sm.luth.se lech@sm.luth.se \\ larserik@sm.luth.se
}

Received: February 1, 2006

Accepted: March 21, 2006

\begin{abstract}
We present a direct proof of a known result that the Hardy operator $H f(x)=$ $\frac{1}{x} \int_{0}^{x} f(t) d t$ in the space $L^{2}=L^{2}(0, \infty)$ can be written as $H=I-U$, where $U$ is a shift operator $\left(U e_{n}=e_{n+1}, n \in \mathbb{Z}\right)$ for some orthonormal basis $\left\{e_{n}\right\}$. The basis $\left\{e_{n}\right\}$ is constructed by using classical Laguerre polynomials. We also explain connections with the Euler differential equation of the first order $y^{\prime}-\frac{1}{x} y=g$ and point out some generalizations to the case with weighted $L_{w}^{2}(a, b)$ spaces.
\end{abstract}

Key words: Hardy inequality, Hardy operator, Laguerre polynomials, isometry, Lebesgue spaces, basis in $L^{2}$ space, weighted $L_{w}^{2}(a, b)$ spaces.

2000 Mathematics Subject Classification: 47B38.

\section{Introduction}

The Hardy averaging operator $H$, defined by $H f(x)=\frac{1}{x} \int_{0}^{x} f(t) d t$, is important in analysis, differential equations and mathematical physics. Therefore a better understanding of the structure of the Hardy operator seems to be important. Moreover, the operator $I-H$ has remarkable mapping properties, i.e., we have the equality

$$
\|(I-H) f\|_{L^{2}}=\|f\|_{L^{2}} \text { for all } f \in L^{2},
$$

Rev. Mat. Complut. 
and this isometry in $L^{2}$ yields also when $H$ is replaced by the dual operator $H^{*}$, defined by $H^{*} f(x)=\int_{x}^{\infty} \frac{f(t)}{t} d t$ (see [1], and for the weighted case [2]).

In section 1 of this paper we will show that if we take the characteristic function of the unit interval $e_{0}=\chi_{(0,1)}$, then the sequence $e_{n}=(I-H)^{n} e_{0}, n=0, \pm 1, \pm 2, \ldots$ forms an orthonormal basis in $L^{2}(0, \infty)$ and therefore the operator $I-H$ is a shift isometry in $L^{2}(0, \infty)$ (see Theorem 1.1). Moreover, the sequence $\left\{e_{n}\right\}$ can be obtained by using some simple transformations from the classical Laguerre polynomials. Theorem 1.1 was earlier proved by Brown-Halmos-Shields [1] but we will give here a direct proof. Our proof is based on an adaptation of known results concerning the Laguerre polynomials.

In section 2 we will discuss connections between the operator $I-H$ and the Euler differential equation

$$
y^{\prime}(x)-\frac{1}{x} y(x)=g(x), \quad y(0)=0, \quad x>0 .
$$

The idea is that if $(I-H) f=g$ or $f=(I-H)^{-1} g$, then $y(x)=\int_{0}^{x} f(t) d t$ is a solution of (2) and therefore (1) implies that, in fact, we have the equality

$$
\left\|y^{\prime}\right\|_{L^{2}}=\|g\|_{L^{2}}
$$

which for the system modelled by (2), can be interpreted as a remarkable precise information between input and output data.

Finally, in section 3 we prove some generalizations of Theorem 1.1) (see Theorems 2.1 and 3.3), point out some consequences of these results and give some concluding remarks.

\section{Laguerre polynomials and a representation formula for the Hardy operator}

Let $L_{n}=L_{n}(x)(n \geq 0)$ be a sequence of Laguerre polynomials (for the information concerning Laguerre polynomials see, e.g., [6, pp. 295-302]). The polynomials $L_{n}$ can be defined as algebraic polynomials such that

(i) $L_{0} \equiv 1, L_{n}(x)$ is a polynomial of degree $n$,

(ii) $\left\{L_{n}\right\}$ is an orthonormal system in $L^{2}=L^{2}(0, \infty)$ with respect to the measure $e^{-x} d x$ :

$$
\int_{0}^{\infty} L_{m}(x) L_{n}(x) e^{-x} d x=\delta_{m, n}
$$

where $\delta_{m, n}$ is the Kronecker delta, that is, $\delta_{m, n}=0$ if $m \neq n$ and $\delta_{m, n}=1$ for $m=n$. 
It is known that $\left\{L_{n}\right\}$ is a basis in $L^{2}(0, \infty)$ with respect to the measure $e^{-x} d x$ (see, e.g., [6, p. 349]). The Laguerre polynomials $L_{n}(x)$ can be expressed by the Rodrigues formula

$$
L_{n}(x)=\frac{e^{x}}{n !} \frac{d^{n}}{x^{n}}\left(x^{n} e^{-x}\right) \quad \text { for } \quad n=0,1,2, \ldots
$$

In particular, $L_{0}(x)=1$ and $L_{1}(x)=1-x$.

Now, we will show how we can construct an orthonormal basis in $L^{2}(0, \infty)$ with the usual measure $d t$ by using the Laguerre polynomials. Since

$$
\begin{aligned}
\delta_{m, n} & =\int_{0}^{\infty} L_{m}(x) L_{n}(x) e^{-x} d x=-\int_{0}^{\infty} L_{m}(x) L_{n}(x) d e^{-x} \\
& =\int_{0}^{1} L_{m}(-\ln t) L_{n}(-\ln t) d t
\end{aligned}
$$

we see that the sequence

$$
f_{n}(t)=L_{n}(-\ln t) \chi_{(0,1)} \quad(n \geq 0)
$$

is an orthonormal system in $L^{2}(0, \infty)$ with the measure $d t$. Moreover, from the completeness of the system $\left\{L_{n}\right\}$ it follows that $\left\{f_{n}\right\}_{n \geq 0}$ is a basis in $L^{2}(0,1)$.

We can also write

$$
\begin{aligned}
\delta_{m, n} & =\int_{0}^{\infty} L_{m}(x) L_{n}(x) e^{-x} d x=\int_{0}^{\infty} \frac{L_{m}(x)}{e^{x}} \frac{L_{n}(x)}{e^{x}} d e^{x} \\
& =\int_{1}^{\infty} \frac{L_{m}(\ln t)}{t} \frac{L_{n}(\ln t)}{t} d t .
\end{aligned}
$$

Hence, we see that the set of functions

$$
e_{n}(t)=-\frac{L_{n}(\ln t)}{t} \chi_{(1, \infty)} \quad(n \geq 0)
$$

(we take here sign "minus" for a later technical reason) is an orthonormal system in $L^{2}(0, \infty)$, which is a basis for $L^{2}(1, \infty)$. Since the sequences $\left\{f_{n}\right\}$ and $\left\{e_{n}\right\}$ have disjoint supports we see that the system

$$
\left\{f_{n}\right\} \cup\left\{e_{n}\right\}
$$

is an orthonormal basis in $L^{2}(0, \infty)$ with the measure $d t$.

To formulate the result let us denote by $U: L^{2} \longrightarrow L^{2}$ the operator defined by the formulas

$$
U f_{0}=e_{0}, \quad U f_{n+1}=f_{n}, \quad U e_{n}=e_{n+1} \quad \text { for } \quad n=0,1,2, \ldots
$$

It is clear that $U$ is a shift isometry in $L^{2}(0, \infty)$.

We are now ready to formulate the main result in this section, namely the following representation formula for the Hardy operator proved already by Brown-HalmosShields [1]. We present here a direct proof. 
Theorem 1.1. The Hardy operator $H f(x)=\frac{1}{x} \int_{0}^{x} f(t) d t$ can be written as

$$
H=I-U
$$

where $U$ is a shift isometry defined by (5).

Proof. We only need to show that the formulas (5) are satisfied for the operator $U=I-H$.

The first equality in formula (5), i.e., the equality $(I-H) f_{0}=e_{0}$, is easy to check by direct calculations since $f_{0}=\chi_{(0,1)}$ and $e_{0}=-\frac{1}{t} \chi_{(1, \infty)}$ (see $(3)$ and $\left.(4)\right)$.

To prove the third equality in (5), i.e., the equality $(I-H) e_{n}=e_{n+1}(n \geq 0)$ we shall use the following properties of the Laguerre polynomials (see [6]):

$$
L_{n}(0)=1, \quad L_{n}^{\prime}(x)-L_{n}(x)=L_{n+1}^{\prime}(x) .
$$

From (6) it follows that

$$
\int_{0}^{x}\left[L_{n}^{\prime}(s)-L_{n}(s)\right] d s=\int_{0}^{x} L_{n+1}^{\prime}(s) d s
$$

and, therefore,

$$
L_{n}(x)-\int_{0}^{x} L_{n}(s) d s=L_{n+1}(x) .
$$

Thus, after the change of variables $x=\ln t, s=\ln \tau$ we have

$$
L_{n}(\ln t)-\int_{1}^{t} \frac{L_{n}(\ln \tau)}{\tau} d \tau=L_{n+1}(\ln t) .
$$

Dividing both parts by $-t$ we see that from (4) it follows that

$$
(I-H) e_{n}=e_{n+1} .
$$

Hence, it only remains to prove that the second equality in formula (5) holds, i.e., that $(I-H) f_{n+1}=f_{n}$ for all $n=0,1,2, \ldots$

To prove this fact let us first prove that from (6) it follows that

$$
\left(\frac{L_{n}(x)}{e^{x}}\right)^{\prime}=\left(\frac{L_{n+1}(x)}{e^{x}}\right)^{\prime}+\frac{L_{n+1}(x)}{e^{x}} .
$$

Indeed, in view of (6) we have

$$
\begin{aligned}
\left(\frac{L_{n}(x)}{e^{x}}\right)^{\prime} & =\frac{L_{n}^{\prime}(x) e^{x}-L_{n}(x) e^{x}}{e^{2 x}}=\frac{L_{n+1}^{\prime}(x) e^{x}}{e^{2 x}} \\
& =\frac{L_{n+1}^{\prime}(x) e^{x}-L_{n+1}(x) e^{x}}{e^{2 x}}+\frac{L_{n+1}(x)}{e^{x}} \\
& =\left(\frac{L_{n+1}(x)}{e^{x}}\right)^{\prime}+\frac{L_{n+1}(x)}{e^{x}}
\end{aligned}
$$


Let us continue the proof of the theorem. From (7) it follows that

$$
\int_{x}^{\infty}\left(\frac{L_{n}(s)}{e^{s}}\right)^{\prime} d s=\int_{x}^{\infty}\left(\frac{L_{n+1}(s)}{e^{s}}\right)^{\prime} d s+\int_{x}^{\infty} \frac{L_{n+1}(s)}{e^{s}} d s,
$$

and, thus,

$$
\frac{L_{n}(x)}{e^{x}}=\frac{L_{n+1}(x)}{e^{x}}-\int_{x}^{\infty} \frac{L_{n+1}(s)}{e^{s}} d s .
$$

After the substitutions $x=-\ln t, s=-\ln \tau(0<t, \tau \leq 1)$ we have

$$
L_{n}(-\ln t)=L_{n+1}(-\ln t)-\frac{1}{t} \int_{0}^{t} L_{n+1}(-\ln \tau) d \tau .
$$

Now putting $t=1$ in (8) and using the fact that $L_{n}(0)=L_{n+1}(0)=1$ (cf. (6)) we find that

$$
\int_{0}^{1} L_{n+1}(-\ln \tau) d \tau=0 \quad(n \geq 0) .
$$

Using (8) and (9) we obtain

$$
L_{n}(-\ln t) \chi_{(0,1)}=L_{n+1}(-\ln t) \chi_{(0,1)}-\frac{1}{t} \int_{0}^{t} L_{n+1}(-\ln \tau) \chi_{(0,1)} d \tau,
$$

which is the equality $(I-H) f_{n+1}=f_{n}$ and so the second equality in the formula (5) is satisfied for the functions $f_{n}=L_{n}(-\ln t) \chi_{(0,1)}$. This means that the proof is complete.

From the theorem it immediately follows that the $L^{2}$-adjoint $(I-H)^{*}$ is equal to $(I-H)^{-1}$.

Corollary 1.2. The operator $(I-H)^{-1}$ is a shift isometry in $L^{2}(0, \infty)$ and, moreover, $(I-H)^{-1}=(I-H)^{*}$ in $L^{2}(0, \infty)$.

\section{On the Euler differential equation}

Let us consider the Euler differential equation of the first order

$$
y^{\prime}(x)-\frac{1}{x} y(x)=g(x), \quad y(0)=0, \quad x>0 .
$$

First we note that if $g \in L^{2}$, then, accordingly to Corollary 1.2, we have that $f=(I-H)^{-1} g \in L^{2}$. Hence, from the Hölder inequality it follows that $\int_{0}^{x} f(t) d t$ exists. If we take $y(x)=\int_{0}^{x} f(t) d t$, then we will have

$$
y^{\prime}-\frac{1}{x} y=(I-H) f=g .
$$


Therefore we see that the solution of the differential equation (10) is given by the formula

and (1) gives

$$
y(x)=\int_{0}^{x}(I-H)^{-1} g(t) d t
$$

$$
\left\|y^{\prime}\right\|_{L^{2}}=\left\|(I-H)^{-1} g\right\|_{L^{2}}=\|g\|_{L^{2}} \quad \text { for any } g \in L^{2} .
$$

Let us now consider the Sobolev space $\dot{W}^{1,2}$ on $(0, \infty)$, i.e., the space of functions $y$ on $(0, \infty)$ with the norm $\|y\|_{\dot{W}^{1,2}}=\left\|y^{\prime}\right\|_{L^{2}}$. (The elements in $\dot{W}^{1,2}$ are functions up to the constants.) Since $(I-H)^{-1}$ maps $L^{2}$ isometrically onto $L^{2}$ and the operator $\operatorname{Pf}(x)=\int_{0}^{x} f(t) d t$ maps isometrically $L^{2}$ onto $\dot{W}^{1,2}$, we find that the equalities (11) and (12) can be interpreted in the following way: the differential operator $D y=y^{\prime}-\frac{1}{x} y$ has a right inverse

$$
(R g)(x)=\int_{0}^{x}(I-H)^{-1} g(t) d t
$$

which maps the space $L^{2}$ isometrically onto the Sobolev space $\dot{W}^{1,2}$.

Naturally appears the question what happens, in a more general situation, when $g$ belongs to some weighted $L^{p}$-space. To formulate the result let us denote by $L_{\alpha}^{p}$ for $\alpha \in \mathbb{R}, p \geq 1$, the space of all functions on $(0, \infty)$ with the norm

$$
\|g\|_{L_{\alpha}^{p}}=\left(\int_{0}^{\infty}\left|\frac{g(t)}{t^{\alpha}}\right|^{p} \frac{d t}{t}\right)^{\frac{1}{p}}
$$

and by $\dot{W}_{\alpha}^{1, p}$ the space of all functions $y$ (up to constants) on $(0, \infty)$ with the norm

$$
\|y\|_{\dot{W}_{\alpha}^{1, p}}=\left\|y^{\prime}\right\|_{L_{\alpha}^{p}}
$$

Theorem 2.1. Let $g \in L_{\alpha}^{p}$ with $p \geq 1$ and $\alpha>-1, \alpha \neq 0$. Then the differential equation (10) has a solution

$$
y(x)=\int_{0}^{x}(I-H)^{-1} g(t) d t \in \dot{W}_{\alpha}^{1, p} .
$$

The operator

$$
(R g)(x)=\int_{0}^{x}(I-H)^{-1} g(t) d t
$$

maps $L_{\alpha}^{p}$ boundedly onto $\dot{W}_{\alpha}^{1, p}$. Moreover, the operator $(I-H)^{-1}$ is given by the formula

$$
(I-H)^{-1} g(x)=g(x)+\int_{0}^{x} g(s) \frac{d s}{s}
$$

for $\alpha>0$ and by the formula

$$
(I-H)^{-1} g(x)=g(x)-\int_{x}^{\infty} g(s) \frac{d s}{s}
$$

for $\alpha \in(-1,0)$. 
Proof. In [3] it was shown (see Remark 5 therein) that if $\alpha>-1, \alpha \neq 0$, then the operator $I-H$ is bounded in $L_{\alpha}^{p}$ and has there a bounded inverse given by the formula (13) for $\alpha>0$ and by the formula (14) for $\alpha \in(-1,0)$. If we consider

$$
f=(I-H)^{-1} g \in L_{\alpha}^{p},
$$

then from the Hölder inequality it follows that the integral $\int_{0}^{x} f(t) d t$ exists. Hence we can take $y(x)=\int_{0}^{x} f(t) d t$ and for such defined $y(x)$ we will obviously have $y^{\prime}-\frac{1}{x} y=$ $(I-H) f=g$.

\section{Generalizations and concluding remarks}

The results in section 1 can obviously be generalized in different directions. Here we will first derive a weighted version of Theorem 1.1). Let $w$ be a positive locally integrable function on $(a, b),-\infty \leq a<b \leq+\infty$, such that

$$
\int_{a}^{b} \omega(t) d t=\infty
$$

Let us consider the weighted space $L_{w}^{2}=L_{w}^{2}(a, b)$ which consists of classes of real-valued measurable functions $f$ defined on $(a, b)$ such that

$$
\|f\|_{L_{w}^{2}}:=\left(\int_{a}^{b} f(x)^{2} w(x) d x\right)^{1 / 2}<\infty .
$$

Theorem 3.1. (i) Suppose that $W(x):=\int_{a}^{x} w(t) d t<\infty$ for any $x \in(a, b)$. Then the operator

$$
H_{w} f(x)=\frac{1}{W(x)} \int_{a}^{x} f(t) w(t) d t
$$

can be written in a form $H_{w}=I-U_{w}$, where $U_{w}$ is a shift isometry in $L_{w}^{2}$.

(ii) Suppose that $\tilde{W}(x):=\int_{x}^{b} w(t) d t<\infty$ for any $x \in(a, b)$. Then the operator

$$
\tilde{H}^{w} f(x)=\frac{1}{\tilde{W}(x)} \int_{x}^{b} f(t) w(t) d t
$$

can be written in a form $\tilde{H}_{w}=I-\tilde{U}_{w}$, where $\tilde{U}_{w}$ is a shift isometry in $L_{w}^{2}$.

Proof. (i) The function $W:(a, b) \rightarrow(0, \infty)$ has the following properties: $W(a)=0$, $W(b)=\infty, W^{\prime}(x)=w(x)>0$ a.e. and is one to one. Moreover,

$$
\begin{aligned}
\left(\int_{0}^{\infty} f(x)^{2} d x\right)^{1 / 2} & =\left(\int_{a}^{b} f(W(t))^{2} W^{\prime}(t) d t\right)^{1 / 2} \\
& =\left(\int_{a}^{b} f(W(t))^{2} w(t) d t\right)^{1 / 2}
\end{aligned}
$$


and, thus, $W$ induces an isometry $T_{w} f(x):=f(W(x))$ between $L^{2}(0, \infty)$ and $L_{w}^{2}(a, b)$. As usual, isometry between spaces induces isometry between operator spaces. In our case we have

$$
\begin{aligned}
H f(W(x)) & =\frac{1}{W(x)} \int_{0}^{W(x)} f(t) d t \\
& =\frac{1}{W(x)} \int_{a}^{x} f(W(s)) w(s) d s=H_{w}\left(T_{w} f\right)(x),
\end{aligned}
$$

so the isometry $T_{w}$ transforms the operator $H$ to the operator $H_{w}$. Therefore, according to Theorem 1.1,

$$
H_{w}=I-U_{w}
$$

where $U_{w}$ is an isometry shift which corresponds to the shift $U$.

(ii) In this case instead of the function $W$ we need to consider the function $\tilde{W}$. The proof is analogous to the proof of (i) so we leave out the details.

Remark 3.2. For the case $a=0$ and $b=\infty$ two proofs of the fact that $H_{\omega}=I-U_{w}$ and $\tilde{H}_{w}=I-\tilde{U}_{w}$, where $U_{w}$ and $\tilde{U}_{w}$ are isometries in $L_{w}^{2}$, can be found in [2] (see also [4, Theorem 5.45]). However, in Theorem 2.1 we proved more (namely that $U_{w}$ and $\tilde{U}_{w}$ are the shift isometries) and the approach above is both easier and put the problem into a more natural frame.

If instead of the isometry $T_{w} f(x)=f(W(x))$ we consider the transformation

$$
S_{w} f(x)=f(W(x)) \sqrt{w(x)},
$$

then it will be induced an isometry between $L^{2}(0, \infty)$ and $L^{2}(a, b)$, which transforms the operator $H$ to the operator

$$
A_{w} f(x)=\frac{\sqrt{w(x)}}{W(x)} \int_{a}^{x} f(t) \sqrt{w(t)} d t
$$

in the case (i) and to the operator

$$
\tilde{A}_{w} f(x)=\frac{\sqrt{w(x)}}{\tilde{W}(x)} \int_{x}^{b} f(t) \sqrt{w(t)} d t
$$

in the case (ii). Therefore, analogously to the Theorem 2.1, we have the following:

Theorem 3.3. (i) If $\int_{a}^{x} w(t) d t<\infty$ for any $x \in(a, b)$, then the operator $I-A_{w}$ is a shift isometry in $L^{2}(a, b)$.

(ii) If $\int_{x}^{b} w(t) d t<\infty$ for any $x \in(a, b)$, then the operator $I-\tilde{A}_{w}$ is a shift isometry in $L^{2}(a, b)$. 
In particular, for the case $(a, b)=(0, \infty)$ and $w(t)=t^{\alpha}$ we obtain the following striking example, which was directly proved and pointed out to us by M. Plum in a personal communication.

Example 3.4. (i) The operator $I-A_{\alpha}$, where

$$
A_{\alpha} f(x)=\frac{\alpha+1}{x^{\frac{\alpha}{2}+1}} \int_{0}^{x} f(t) t^{\frac{\alpha}{2}} d t
$$

is a shift isometry in $L^{2}(0, \infty)$ for $\alpha>-1$.

(ii) Analogously the operator $I-\tilde{A}_{\alpha}$, where

$$
\tilde{A}_{\alpha} f(x)=-\frac{\alpha+1}{x^{\frac{\alpha}{2}+1}} \int_{x}^{\infty} f(t) t^{\frac{\alpha}{2}} d t
$$

is a shift isometry in $L^{2}(0, \infty)$ for $\alpha<-1$.

Remark 3.5. Example 3.4 shows that there are scales of operators $A_{\alpha}$ and $\tilde{A}_{\alpha}$ satisfying (1) instead of $H$ and this fact and all other results in this paper contributes to the understanding of an open Problem 3 in [4, p. 299].

Remark 3.6. In this paper all results are equipped with $L^{2}$, or weighted $L^{2}$ spaces. However, our original interest in this subject was connected with the following result for weighted $L^{p}$ spaces (see [3] and also [4, Prop. 5.38]):

$$
\text { Let } f \in L_{\alpha}^{p} \text { with } p \geq 1 \text { and } \alpha>-1, \alpha \neq 0 \text {. Then }
$$

$$
\int_{0}^{\infty}\left|\frac{f(x)-\frac{1}{x} \int_{0}^{x} f(t) d t}{x^{\alpha}}\right|^{p} \frac{d x}{x} \approx \int_{0}^{\infty}\left|\frac{f(x)}{x^{\alpha}}\right|^{p} \frac{d x}{x}
$$

with the constant of equivalence independent of $f$.

Many questions are of interest in this connection, e.g., to find the sharp constants in (16).

Acknowledgements. We thank M. Plum for early pointing out Example 3.4 and P. P. Zabreiko for valuable advices and support.

\section{References}

[1] A. Brown, P. R. Halmos, and A. L. Shields, Cesàro operators, Acta Sci. Math. (Szeged) 26 (1965), 125-137.

[2] N. Kaiblinger, L. Maligranda, and L.-E. Persson, Norms in weighted $L^{2}$-spaces and Hardy operators, Function Spaces, The Fifth Conference (Poznań, 1998), Lecture Notes in Pure and Appl. Math., vol. 213, Dekker, New York, 2000, pp. 205-216. 
[3] N. Krugljak, L. Maligranda, and L. E. Persson, On an elementary approach to the fractional Hardy inequality, Proc. Amer. Math. Soc. 128 (2000), no. 3, 727-734.

[4] A. Kufner and L.-E. Persson, Weighted inequalities of Hardy type, World Scientific Publishing Co. Inc., River Edge, NJ, 2003.

[5] B. Opic and A. Kufner, Hardy-type inequalities, Pitman Research Notes in Mathematics Series, vol. 219, Longman Scientific \& Technical, Harlow, 1990

[6] G. Sansone, Orthogonal functions, rev. English ed., Dover Publications Inc., New York, 1991. 